\title{
TITLE:
}

\section{Charged colloidal system: Small ion distribution and effective interaction}

\author{
$\operatorname{AUTHOR}(\mathrm{S})$ :
}

Ikeda, Ichiro; Matsumoto, Mitsuhiro

\section{CITATION:}

Ikeda, Ichiro ...[et al]. Charged colloidal system: Small ion distribution and effective interaction. Colloids and Surfaces A: Physicochemical and Engineering Aspects 2014, 440: 34-41

ISSUE DATE:

2014-01-05

URL:

http://hdl.handle.net/2433/180110

\section{RIGHT:}

(c) 2012 Elsevier B.V.; この論文は出版社版でありません。引用の際には 出版社版をご確認ご利用ください。; This is not the published version. Please cite only the published version. 


\title{
Charged Colloidal System: Small Ion Distribution and Effective Interaction
}

\author{
Ichiro Ikeda ${ }^{\mathrm{a}, *}$, Mitsuhiro Matsumoto ${ }^{\mathrm{a}, \mathrm{b}}$ \\ ${ }^{a}$ Department of Mechanical Engineering and Science, \\ Kyoto University, Kyoto 606-8501, Japan \\ ${ }^{b}$ Advanced Research Institute of Fluid Science and Engineering, \\ Kyoto University, Kyoto 615-8530, Japan
}

\begin{abstract}
We applied a molecular dynamics simulation technique to charged colloidal systems in order to investigate small ion distribution in thermal equilibrium and to evaluate the effective interaction among macroions. For a system containing a single macroion, screening by counter ions was evaluated. The screening becomes more complete for larger macroion charge. In spite of the large inhomogeneity, the chemical potential of small ions is constant for the entire system. Using a thermodynamic integral method, the effective potential (Helmholtz free energy) for two-macroion systems was evaluated as a function of macroion separation distance, and found to be repulsive under normal conditions. Many-body effect is also discussed by comparing the effective potentials for two- and three-macroion systems.
\end{abstract}

Keywords: Charged colloid, Effective potential, Molecular dynamics simulation, Many-body effect

\footnotetext{
${ }^{*}$ Corresponding author. Tel.:+81 75 7535217; fax:+81 757717286

Email address: ikeda.ichirou.85e@st.kyoto-u.ac.jp (Ichiro
} Ikeda) 


\section{Introduction}

Charged colloidal systems (latexes, metal nanoparticles, proteins, etc.) have been widely studied in various fields of science and engineering. Among many interesting phenomena, they have an ordered or crystalline state under some conditions to show iridescence. It is conventionally explained with the DLVO theory[1], which takes account of repulsion due to the overlap of electric double layers and the van der Waals attraction. When the salt concentration is very low, the screening by counter ions is incomplete, causing strong repulsive interaction. The crystallization is then the Alder type transition[2, 3]. Upon adding more salt, however, the crystalline structure disappears[4], contrary to the theoretical prediction of entrapment in a potential minimum. Sogami and Ise has proposed a model[5] that explains the phenomena with electrostatic interaction only. He analytically calculated the Gibbs free energy of the system and showed that electrostatic attraction can exist between macroions of same charge. It has been long controversial, but some recent experiments seem to support the concept of electrostatic attraction[6]. Another theoretical aspect is the treatment of the Poisson-Boltzmann equation. Conventionally a linearization approximation has been adopted with implicit assumption of weak interactions. Akiyama et al.[7] recently reported based on an integral equation approach (an OrnsteinZernike equation combined with hypernetted-chain closure) without the linearization that an attractive interaction between macromolecules appears in some range of salt concentration.

In this paper, we adopt a molecular simulation approach to investigate charged colloidal systems. Our main objective is to estimate the effective interaction between two macroion of same charge. For that purpose, we carry out a series of molecular dynamics simulations for small ions with macroions of fixed positions. With a similar approach, we also investigate small ion distribution around a single macroion to see the screening, in comparison with the linearization approximation. Because main purpose is the comparison to the theoretical solutions, many simulations are done for salt-free systems to shorten the computation time.

\begin{tabular}{|c|c|}
\hline \multicolumn{2}{|c|}{ Nomenclature } \\
\hline$A$ & free energy or effective potential $(\mathrm{J})$ \\
\hline$e$ & elementary charge $(\mathrm{C})$ \\
\hline$F$ & force $(\mathrm{N})$ \\
\hline$k_{\mathrm{B}}$ & Boltzmann constant $(\mathrm{J} / \mathrm{K})$ \\
\hline$l$ & distance between macroion surface and a small ion $(\mathrm{m})$ \\
\hline$l_{B}$ & the Bjerrum length (m) \\
\hline$m$ & mass of a small ion $(\mathrm{kg})$ \\
\hline$n$ & number density $\left(\mathrm{m}^{-3}\right)$ \\
\hline$N$ & number of particles $(-)$ \\
\hline$p$ & momentum of small ion $\left(\mathrm{kg} \cdot \mathrm{m} / \mathrm{s}^{2}\right)$ \\
\hline$P$ & pressure $(\mathrm{Pa})$ \\
\hline$q$ & charge of a small ion $(\mathrm{C})$ \\
\hline$Q_{N}$ & partition function $(-)$ \\
\hline$r$ & distance from the center of a macroion $(\mathrm{m})$ \\
\hline$R$ & distance between macroions $(\mathrm{m})$ \\
\hline$R_{a}$ & macroion radius $(\mathrm{m})$ \\
\hline$T$ & temperature $(\mathrm{K})$ \\
\hline$U$ & potential energy $(\mathrm{J})$ \\
\hline$V$ & volume $\left(\mathrm{m}^{3}\right)$ \\
\hline$Z$ & a macroion charge (valence) $(-)$ \\
\hline \multicolumn{2}{|c|}{ Greek letters } \\
\hline$\epsilon$ & Lennard-Jones energy parameter $(\mathrm{J})$ \\
\hline$\epsilon_{\mathrm{w}}$ & permittivity of water $(\mathrm{F} / \mathrm{m})$ \\
\hline$\phi$ & interaction potential between particles $(\mathrm{J})$ \\
\hline$\lambda$ & Debye screening length $(\mathrm{m})$ \\
\hline$\Lambda$ & thermal de Broglie wavelength (m) \\
\hline$\mu$ & chemical potential $(\mathrm{J})$ \\
\hline$\sigma$ & Lennard-Jones size parameter $(\mathrm{m})$ \\
\hline \multicolumn{2}{|c|}{ superscript } \\
\hline c & Coulombic \\
\hline & excess term \\
\hline id & ideal gas term \\
\hline LJ & Lennard-Jones \\
\hline PB & solution of (linearized) Poisson-Boltzmann equation \\
\hline \multicolumn{2}{|c|}{ subscript } \\
\hline & effective charge \\
\hline$i$ & $i$-th species of small ions \\
\hline $\mathrm{m}$ & macroion \\
\hline run & running sum \\
\hline $\mathrm{s}$ & small ion \\
\hline 0 & value averaged over the simulation cell \\
\hline
\end{tabular}




\section{Simulation Method}

We adopted a simple canonical ensemble molecular dynamics (MD) simulation for small ions, assuming that solvent (water) is just a medium with appropriate permittivity. A Brownian dynamics (BD) technique [8] is another candidate, but both methods should give the same static properties (ion distributions and effective force on macroions) at equilibrium states.

We assume that the potential energy of the system is a sum of two-body functions,

$$
U=\sum_{i, j}\left[\phi_{\mathrm{ss}}^{\mathrm{c}}\left(r_{i j}\right)+\phi_{\mathrm{ss}}^{\mathrm{LJ}}\left(r_{i j}\right)\right]+\sum_{i, k}\left[\phi_{\mathrm{sm}}^{\mathrm{c}}\left(r_{i k}\right)+\phi_{\mathrm{sm}}^{\mathrm{LJ}}\left(r_{i k}\right)\right]+\sum_{k, l} \phi_{\mathrm{mm}}^{\mathrm{c}}\left(r_{k l}\right)(1)
$$

where $i$ and $j$ represent small ions, $k$ and $l$ represent macroions. The subscript $\mathrm{s}$ and $\mathrm{m}$ correspond to small ions and macroions, respectively. The Coulombic interaction between $i$-th (small or macro) ion with charge $q_{i}$ and $j$-th with $q_{j}$ is

$$
\phi^{\mathrm{c}}\left(r_{i j}\right)=\frac{1}{4 \pi \epsilon_{\mathrm{w}}} \frac{q_{i} q_{j}}{r_{i j}}
$$

where $\epsilon_{\mathrm{W}}=80.4 \epsilon_{0}$ is the permittivity of water at room temperature $\left(\epsilon_{0}\right.$ : vacuum permittivity).

The short-range Lennard-Jones (LJ) type interaction is assumed only for $\mathrm{s}-\mathrm{s}$ and $\mathrm{s}-\mathrm{m}$. The primitive model has used to express the potential of the system with the macroion and small ions in some past literatures[13, 14, 15, 20]. In primitive model the potential of the system is assumed to the Coulombic potential and the hard core potential. We use the LJ type interaction instead of the hard core potential to be closer to reality that the macroion is the cluster of the small molecule.

For the interaction between small ions, we use a conventional LJ (12-6) potential depending on the particle distance $r$ as

$$
\phi_{\mathrm{ss}}^{\mathrm{LJ}}\left(r_{i j}\right)=4 \epsilon_{i j}\left[\left(\frac{\sigma_{i j}}{r_{i j}}\right)^{12}-\left(\frac{\sigma_{i j}}{r_{i j}}\right)^{6}\right]
$$

where $\epsilon_{i j}$ and $\sigma_{i j}$ are the LJ parameters. Since macroions are sufficiently larger than small ions, the LJ interaction between a small ion and a macroion is given by integrating the LJ (12-6) potential in a semi-infinite space, leading to

$$
\phi_{\mathrm{sm}}^{\mathrm{LJ}}(l)=8 \pi \epsilon_{\mathrm{m}} n_{\mathrm{m}} \sigma_{\mathrm{m}}^{3}\left[\frac{1}{90}\left(\frac{\sigma_{\mathrm{m}}}{l}\right)^{9}-\frac{1}{12}\left(\frac{\sigma_{\mathrm{m}}}{l}\right)^{3}\right]
$$

as a function of the distance $l$ from the macroion surface, where $\epsilon_{\mathrm{m}}$ and $\sigma_{\mathrm{m}}$ are the LJ parameters for macroions, and $n_{\mathrm{m}}$ is the number density inside the macroion.

In this study, we assume that the macroions (colloid particles) are polyacrylic acid (PAA) anions with constant charge $-Z e(e$ : elementary charge) being uniformly distributed in each sphere of radius $R_{a}$. Counter ions are then cations and sodium ions $\left(\mathrm{Na}^{+}\right)$are assumed, while chloride ions $\left(\mathrm{Cl}^{-}\right)$are used for coions. Parameters are listed in Table 1. The Lorentz-Berthelot combination rule is assumed for LJ parameters between different species:

$$
\left\{\begin{aligned}
\epsilon_{i j} & =\sqrt{\epsilon_{i} \epsilon_{j}} \\
\sigma_{i j} & =\frac{\sigma_{i}+\sigma_{j}}{2}
\end{aligned}\right.
$$

Table 1: Parameters used in the simulation.

\begin{tabular}{c|cc} 
& \multicolumn{2}{c}{$(*)$ except for otherwise stated. } \\
\hline \hline & Cation $\left(\mathrm{Na}^{+}\right)$ & Anion $\left(\mathrm{Cl}^{-}\right)$ \\
\hline$\epsilon / k_{\mathrm{B}}[\mathrm{K}]$ & 32.8 & 93.3 \\
$\sigma[\mathrm{nm}]$ & 0.282 & 0.3542 \\
$m[\mathrm{~g} / \mathrm{mol}]$ & 22.99 & 35.453 \\
$q[\mathrm{C}]$ & $e\left(^{*}\right)$ & $-e$ \\
\hline \hline \multicolumn{2}{|c}{} \\
\hline \hline \multicolumn{2}{|c}{ Macroion (PAA anion) } \\
\hline$\epsilon_{\mathrm{m}} / k_{\mathrm{B}}[\mathrm{K}]$ & 32.8 \\
$\sigma_{\mathrm{m}}[\mathrm{nm}]$ & 0.282 \\
$Z$ & $-100 \sim-10000$ \\
$\operatorname{Radius~} R_{a}[\mathrm{~nm}]$ & 10 \\
$n_{\mathrm{m}}\left[(\mathrm{nm})^{-3}\right]$ & 1.910 \\
\hline \hline
\end{tabular}

We carried out a series of MD simulations for small ions confined in a rectangular parallelepiped cell of constant volume $V$. The position of macroion(s) is fixed, so that the macroions exert an external force field on the small ions. Reflective boundary conditions are utilized instead of the usual periodic boundaries; if we assume periodic boundary conditions, ions in the neighboring image cell would affect the effective force on macroions. Thus we sum up the interactions for all ion pairs without truncation.

The time step for integration is $5 \mathrm{fs}$. The temperature is evaluated from the mean kinetic energy of small ions, and kept constant at room temperature $\left(20^{\circ} \mathrm{C}\right)$ with the velocity scaling technique. After equilibrating the system at a given condition, data are accumulated typically during 10,000 steps for later analyses.

\section{Single Macroion Case}

As a first step, we investigate small ion distributions around a single macroion to see how counter ions screen the macroion charge. Most of the simulations were done in a cubic cell of $V=50 \mathrm{~nm} \times 50 \mathrm{~nm} \times 50 \mathrm{~nm}$. In this case the volume fraction is 0.034 .

\subsection{Small ion distribution}

An example of counter ion distribution in equilibrium is shown in Fig. 1. By averaging data over 10,000 steps, we obtained small ion concentrations as functions of distance $r$ from the center of the macroion. Examples for $Z=-1000$ case are shown in Fig. 2 for (a) a salt-free system, and (b) a system with 500 salt ion pairs where totally 1,500 counter ions and 500 coions exist.

As expected, the counter ions $\left(\mathrm{Na}^{+}\right)$gather around the macroion and screen its charge, while the co-ions $\left(\mathrm{Cl}^{-}\right)$are expelled from it. The concentration of each species seems constant beyond $20 \mathrm{~nm}$. 


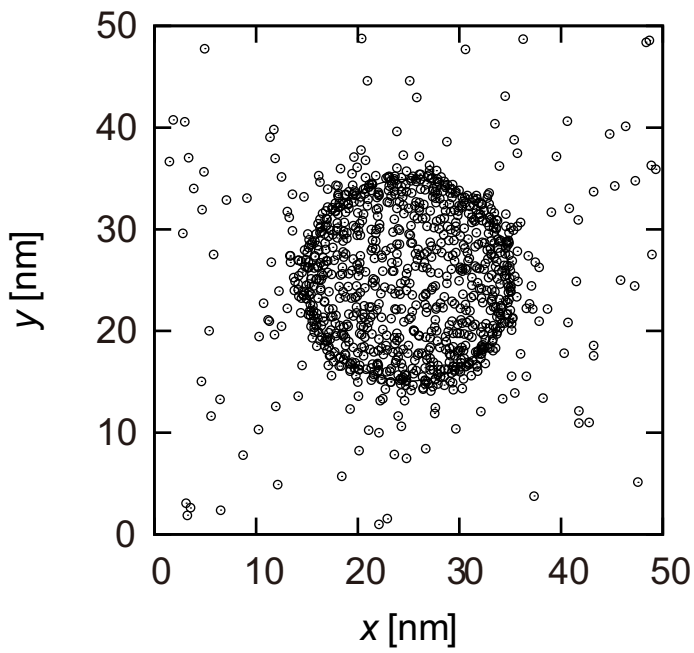

Figure 1: Example of counter ion distribution around a macroion; a snapshot projected to the $x-y$ plane, for the case of $Z=-1000$.

\subsection{Effective charge}

To examine the charge screening by counter ions, we define a running sum charge at the distance $r$ from the center of the macroion as the running sum charge at $r=\lambda$,

$$
Z_{\mathrm{run}}(r)=Z+\sum_{i} \frac{q_{i}}{e} \int_{R_{a}}^{r} n_{i}\left(r^{\prime}\right) 4 \pi r^{\prime 2} d r^{\prime}
$$

where $n_{i}(r)$ is the concentration (number density) of $i$-th species, and the sum is taken for all small ion species.

Examples for salt-free systems are shown in Fig. 3. When the macroion cahrge $Z$ is small, the running sum charge exponentially decreases with $r$. For the case of large $Z$, however, the charge screening is more evident; a large portion of the intrinsic macroion charge is neutralized at the Debye screening length, define as

$$
\lambda=\left[\frac{1}{\epsilon_{\mathrm{w}} k_{B} T} \sum_{i} q_{i}^{2} n_{i 0}\right]^{-\frac{1}{2}}
$$

where $n_{i 0}$ is the average concentration of $i$-th species. Thus we define the effective charge of a macroion $Z_{\mathrm{eff}}$ as

$$
Z_{\text {eff }}=Z_{\text {run }}(\lambda)
$$

Figure 4 shows how the effective charge increases with $Z$. For comparison of the analytical solution we show the effective charge defined by Aubouy et al as following equation[25]:

$$
Z_{\mathrm{eff}} \frac{l_{\mathrm{B}}}{R_{a}}=4 \frac{R_{a}}{\lambda} t_{z}+2\left(5-\frac{t_{z}^{4}+3}{t_{z}^{2}+1}\right) t_{z}
$$

where $l_{\mathrm{B}}=e^{2} /\left(\epsilon_{\mathrm{w}} k_{\mathrm{B}} T\right)$ is the Bjerrum length and $t_{z}$ is defined as folloeing equation using function $T$ :

$$
\begin{aligned}
& t_{z}=T\left(\frac{Z l_{B} / R_{a}}{2 R_{a} / \lambda+2}\right) \\
& T(x)=\frac{\sqrt{1+x^{2}}-1}{x}
\end{aligned}
$$
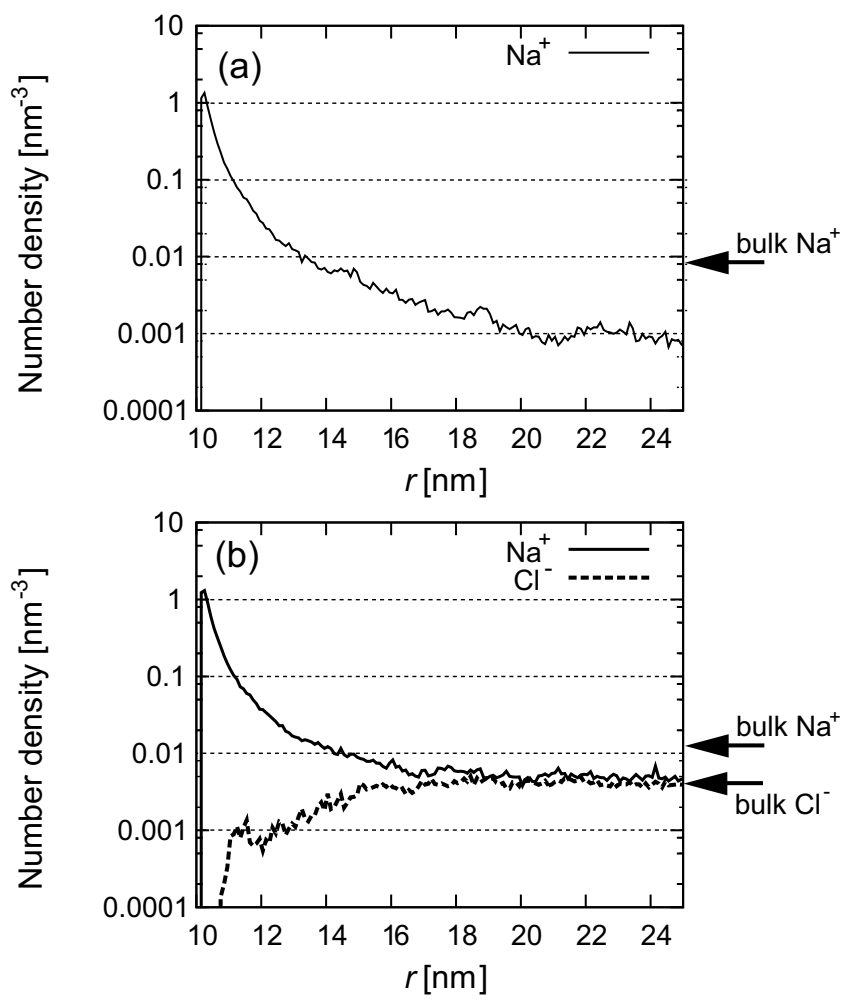

Figure 2: Example of small ion concentration around a macroion with $Z=$ -1000 in a $50 \times 50 \times 50\left(\mathrm{~nm}^{3}\right)$ cell; (a) A salt-free case and (b) a case with 500 salt ion pairs. The arrows indicate the average (or "bulk") concentration.

As the macroion charge increases, the screening becomes more complete and the effective charge saturates at the charge $Z=-5000$. Yamanaka et al empirically shows the power relation betweeen the effective surface charge density and the analytical surface charge density[16]. There are the difference of the charge and surface charge. The macroion radius is fixed in our simulation, so the comparison is capable to be done. However in our simulation the power relation is not seen.

Effective charge of colloids have been simulated by many people with various numerical methods and various definitions. Alexander et al defined the effective charge as the charge in the Wigner-Seitz (WS) sphere and caluculates by the non liniar Poisson-Boltzmann equation[17]. While Bocquet et al gained the effective charge from the solution of the liniarized Poisson Boltzmann (LPB) equation closing to that of Poisson Boltzmann equation far from macroion surface and proposed that the effective charge saturates[18]. Belloni showed that all effective charge gained by Bjerrum length, osmotic pressure, selfdiffusion coefficient and the WS sphere are of the same order of magnitude[26]. We define the effective charge as the charge in the Deby length from the macroion surface. To evaluate the adequacy of this definition, we compared the electric potential gained from the simulation results and the LPB solution. These electric potential are almost same far from macroion surface. We think that effective charge from Debye length almost corresponds to the effective charge from LPB solution. 

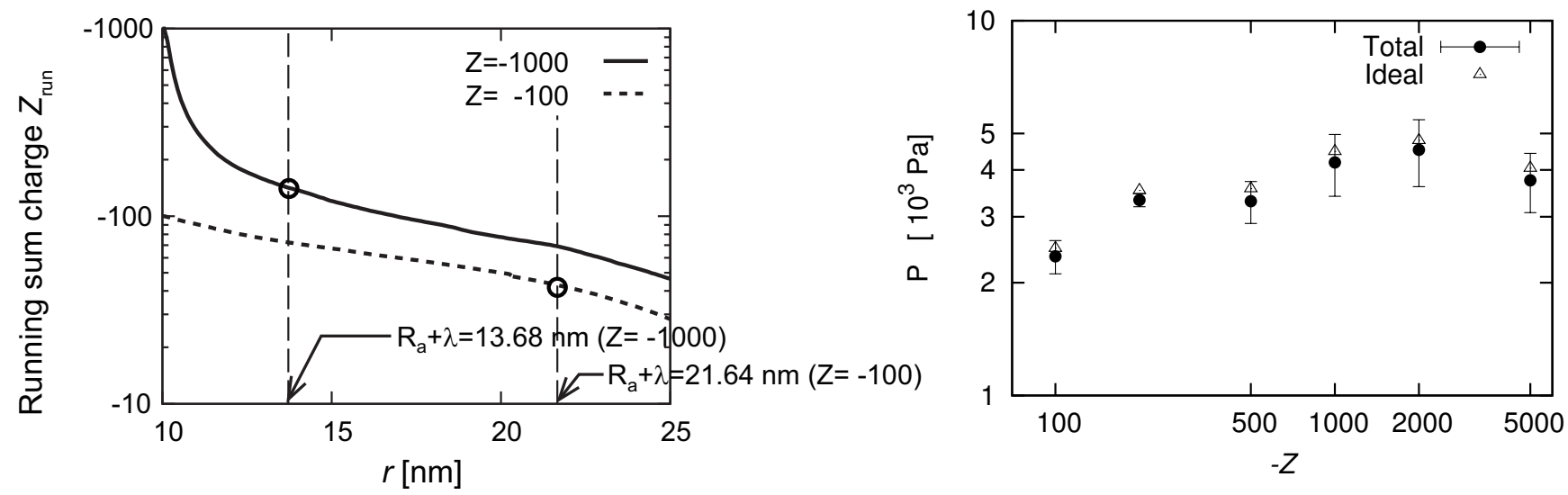

Figure 3: Running sum charge for salt-free systems. Vertical dashed lines indicate the Debye screening length.

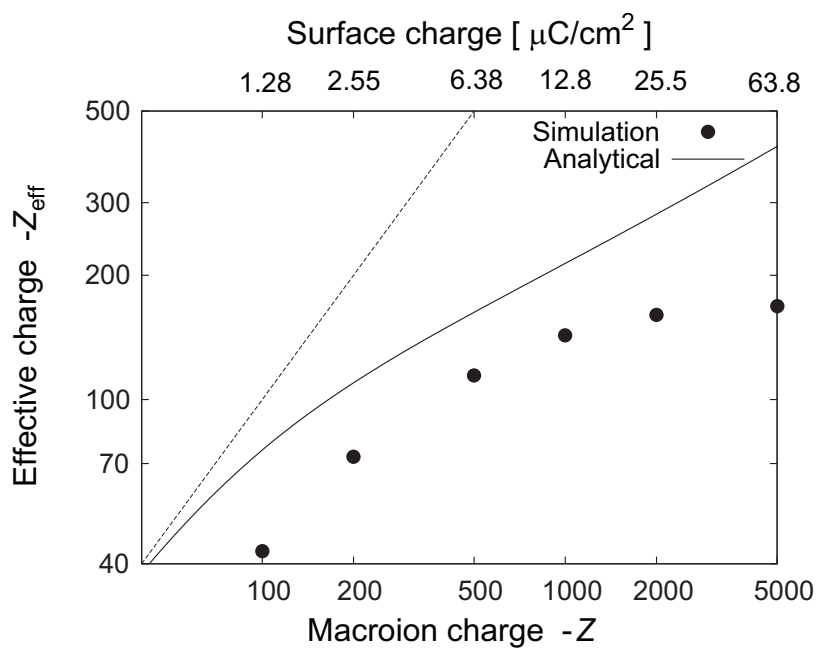

Figure 6: Pressure for salt-free systems; (filled circles) the total pressure, (open triangles) ideal gas term of the pressure. Pressure saturates with the macroion charge $Z$ increasing.

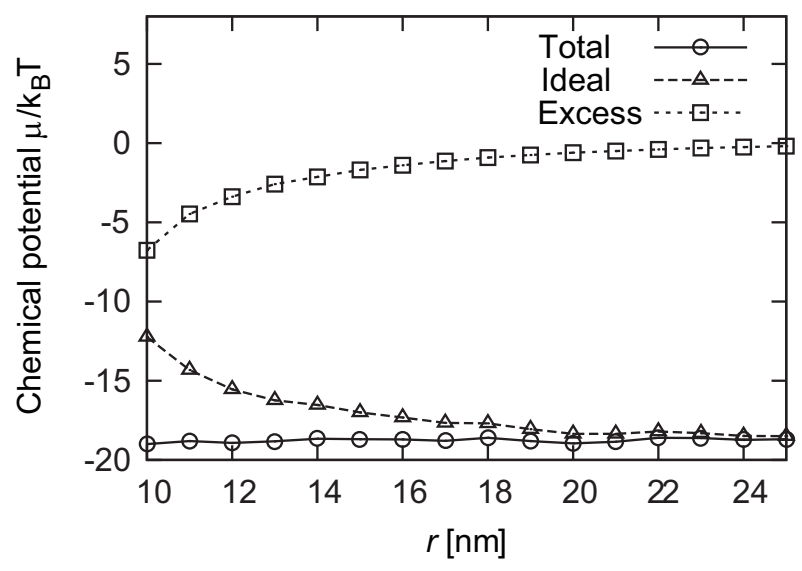

Figure 4: Effective charge gained at the Debye length from the macroion surface. (filled circles) simulation, (line) analytical solution defined by the Aubouy et al[25]. As the macroion charge $Z$ increases, the effective charge $Z_{\text {eff }}$ deviates from $Z$ (shown with dotted line) due to charge screening.

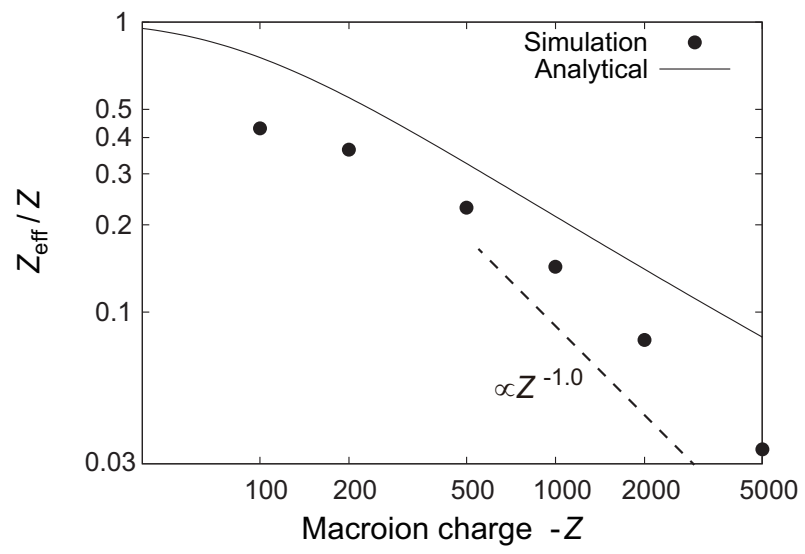

Figure 5: Screening ratio gained at the Debye length from the macroion surface.(filled circles) simulation, (line) analytical solution defined by the Aubouy et al[25]. A inverse proportion law (shown with dotted line) seems to hold for large $Z$ region.

Figure 7: Local chemical potential of counter ions for a salt-free system with a macroion of $Z=-1000$ in a $50 \mathrm{~nm} \times 50 \mathrm{~nm} \times 50 \mathrm{~nm}$ cell.

The osmotic pressure has simulated for the comparison of experimental data. The osmotic pressure in the solution has gained from the densities of small ions at the WignerSeitz sphere[17, 18]. To investigate the relation between the macroion charge and the pressure, we define the pressure $P$ near the box wall as the average local pressure in the range of $r=20 \sim 25$ as the following equation:

$$
P=\frac{1}{3 V}\left(\left\langle\sum_{i=1}^{N} \frac{\mathbf{p}_{i}^{2}}{m_{i}}\right\rangle_{r=20 \sim 25}-\left\langle\sum_{i=1}^{N} \mathbf{r}_{i} \cdot \frac{\partial U}{\partial \mathbf{r}_{i}}\right\rangle_{r=20 \sim 25}\right)
$$

where $\mathbf{p}_{i}$ is the momentum of $\mathrm{i}$-th small ion. The time step average is done over 10,000 steps. As showen in Fig. 6, at the macroion charge $Z=-1000$ pressure takes the max value. The pressure is also seen to saturate as same as the effective charge. The effect of ideal gas term influences over nine-tenths of the osmotic pressure. The virial pressure is negligible as the past literatures pointing. 


\subsection{Chemical potential}

The distribution of small ions in the system is thus very inhomogeneous. Can we still expect the conventional conditions for homogeneous chemical potential for systems in thermal equilibrium? We investigate the local chemical potential,

$$
\begin{aligned}
\mu & \simeq A(V, T, N+1)-A(V, T, N) \\
& =\mu^{\text {id }}+\mu^{\mathrm{ex}}
\end{aligned}
$$

where $A$ is the Helmholtz free energy of the $N$ particle system at temperature $T$. The ideal gas part is then obtained as

$$
\mu^{\text {id }}=-k_{\mathrm{B}} T \log \frac{V}{\Lambda^{3}(N+1)}
$$

where $\Lambda$ is the thermal de Broglie wavelength. The excess part is evaluated via the particle insertion method $[9,10]$

$$
\mu^{\mathrm{ex}}=-k_{\mathrm{B}} T \log \left\langle\exp \left[-\frac{U^{N+1}\left(r^{N}\right)}{k_{\mathrm{B}} T}\right]\right\rangle_{N},
$$

through averaging the Boltzmann factor of energy $U^{N+1}$, which is the potential energy increment by inserting a ghost particle. For a insert particle charge neutrality of the total system is broken. So the individual small ion chemical potential is overestimated. Svensson et al gained the ion chemical potential by inserting the ion pair without breaking the electroneutrality[24]. In our simulation small ion chemical potential is overestimated for the breaking of the charge neutrality.

An example is shown in Fig. 7, where the chemical potential of counter ion (cation) in a salt-free system is evaluated as a function of distance $r$ from the center of the macroion. In the vicinity of the macroion, the increase of $\mu^{\text {id }}$ due to the counter ion condensation and the decrease of $\mu^{\mathrm{ex}}$ due to the stronger interaction with the macroion cancel each other, leading to the constant $\mu$. Thus the homogeneity of chemical potential is confirmed.

\section{Two Macroions Case}

Now we evaluate an effective interaction between two macroions of the same charge, which is the main target of this study. It is rather straightforward to measure the force acting on each macroion, although it largely fluctuates. From the timeaveraged force, we can estimate the effective potential, or the free energy.

\subsection{Thermodynamic integration method}

Suppose a Helmholtz free energy $A(V, T ; \eta)$ as a function of some external parameter $\eta$ in general. The difference $\Delta A$ by changing the parameter from $\eta_{0}$ to $\eta_{1}$ is then expressed as

$$
\begin{aligned}
\Delta A & =A\left(\eta_{1}\right)-A\left(\eta_{0}\right) \\
& =\int_{\eta_{0}}^{\eta_{1}} \frac{\partial A(\eta)}{\partial \eta} d \eta
\end{aligned}
$$

Since $A$ is related to the partition function $Q_{N}$,

$$
A=-k_{\mathrm{B}} T \ln Q_{N}(V, T ; \eta),
$$
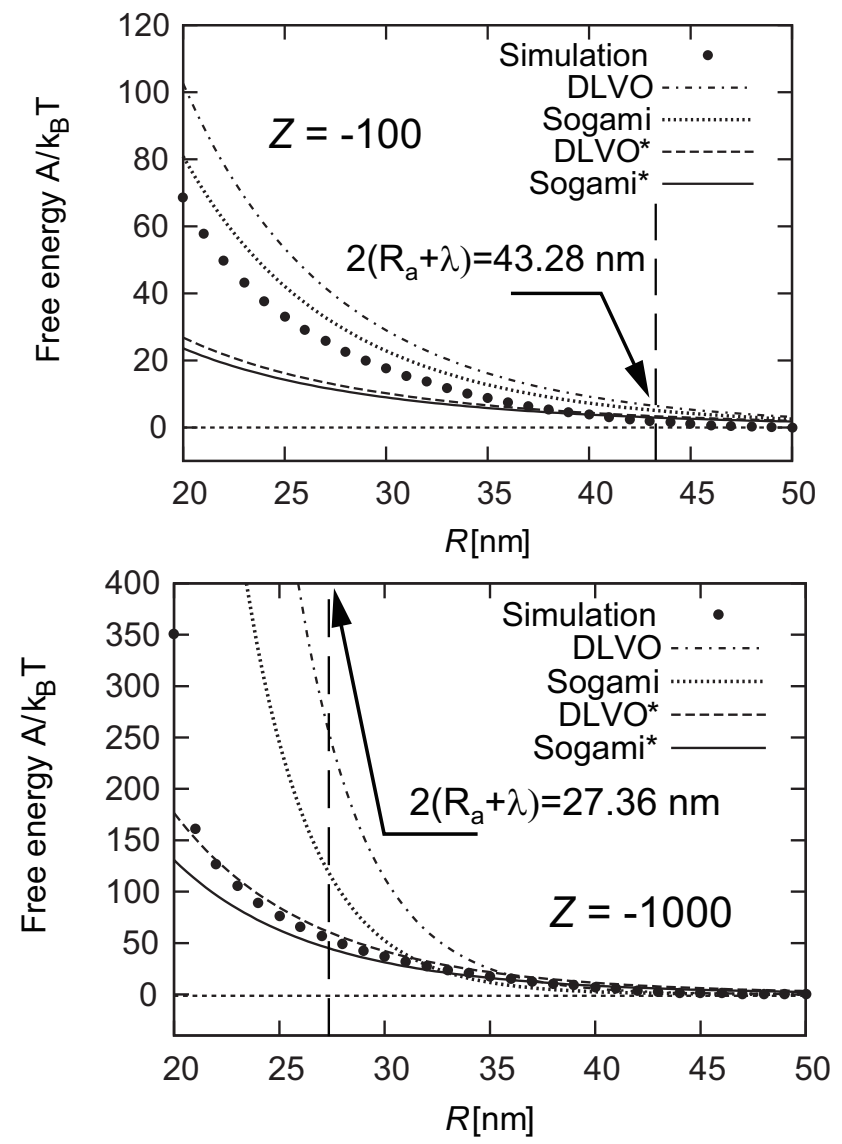

Figure 8: Evaluated effective potential between two macroions, compared with DLVO theory and Sogami model; a salt-free system in a $100 \mathrm{~nm} \times 50 \mathrm{~nm} \times 50 \mathrm{~nm}$ cell. The Debye screening length is indicated by the vertical dashed line. The superscript $*$ shows the value gained from the effective charge.

we can obtain the following integration (thermodynamic integration [11]) for $\Delta A$ :

$$
\begin{aligned}
\Delta A & =\int_{\eta_{0}}^{\eta_{1}}-\frac{k_{\mathrm{B}} T}{Q_{N}} \frac{\partial Q_{N}(\eta)}{\partial \eta} d \eta \\
& =\int_{\eta_{0}}^{\eta_{1}}\left\langle\frac{\partial U_{N}(\eta)}{\partial \eta}\right\rangle_{\eta} d \eta
\end{aligned}
$$

When we choose the distance between the two macroions $R$ as $\eta$, we can evaluate $\Delta A$ via the integration of "mean force" $F$ :

$$
\begin{aligned}
A\left(R_{1}\right)-A\left(R_{0}\right) & =\int_{R_{0}}^{R_{1}}\left\langle\frac{\partial U_{N}(R)}{\partial R}\right\rangle_{R} d R \\
& =-\int_{R_{0}}^{R_{1}}\langle F(R)\rangle_{R} d R
\end{aligned}
$$

\subsection{Effective potential between two macroions}

An example is shown in Fig. 8 for salt-free cases (macroion charge $Z=-100$ and -1000$)$. We found that the interaction is repulsive in general. For comparison, two model potentials are also shown:

\section{$(\mathrm{DLVO}[1]) \quad U_{\mathrm{DLVO}}(R)$}


Table 2: Simulation conditions for Fig. 9

\begin{tabular}{cccc}
\hline \hline & Case 1 & Case 2 & Case 3 \\
\hline Volume $\left[(\mathrm{nm})^{3}\right]$ & $100 \times 50 \times 50$ & $200 \times 100 \times 100$ & $200 \times 100 \times 100$ \\
Number of cations & 200 & 200 & 1000 \\
Number of anions & 0 & 0 & 800 \\
Debye length $\lambda[\mathrm{nm}]$ & 11.64 & 33.43 & 11.14 \\
Volume fraction & 0.034 & 0.0042 & 0.0042 \\
\hline \hline
\end{tabular}

$$
=\frac{Z^{2} e^{2}}{4 \pi \epsilon_{\mathrm{w}}\left(1+R_{a} / \lambda\right)^{2}} \frac{1}{R} \exp \left[\frac{2 R_{a}-R}{\lambda}\right]
$$

$$
\begin{aligned}
& \text { (Sogami [6]) } \quad U_{\text {Sogami }}^{\mathrm{F}}(R) \\
& \quad=\frac{Z^{2} e^{2} \sinh ^{2}\left(R_{a} / \lambda\right)}{4 \pi \epsilon_{\mathrm{w}}\left(R_{a} / \lambda\right)^{2}} \frac{1}{R} \exp \left[-\frac{R}{\lambda}\right]
\end{aligned}
$$

Model potentials are evaluated for the net charge and the effective charge. In evaluating for the effective charge the Deby length $\lambda$ is also modified to keep the charge neutrality. Note that, for the Sogami model, $U^{\mathrm{F}}$ (Helmholtz free energy) is compared instead of $U^{\mathrm{G}}$, because $\Delta A$ is evaluated with the constant volume condition.

At the macroion charge $Z=-100$, the obtained effective potential is close to the theoretical model evaluated the net charge. While at the macroion charge $Z=-1000$, the obtained effective potential is very close to each model evaluated effective charge in the large distances $R$ except for $R=20$. The modification of the model potential by the effective charge gained from the Debye length is appropriate in the large macroion charge, but is not appropriate in the small charge for underestimateing the effective charge.

\subsection{Volume dependence}

Conventional models, either the DLVO or the Sogami, in principle assume an infinite system, where small ions distribute in a space of macroscopic scale. In our simulation, on the other hand, ions are confined in a finite cell of volume $V$ without periodic boundary conditions, partly because we cannot treat infinite number of ions and we want to exclude effects from "image" cells.

Thus we have to check how the system size affects the simulation results. Shown in Fig. 9 are the comparison of effective potential between systems with different volume; the Case 1 is the result for the reference system with $V=100 \mathrm{~nm} \times 50 \mathrm{~nm} \times 50 \mathrm{~nm}$, while the Case 2 is for $200 \mathrm{~nm} \times 100 \mathrm{~nm} \times 100 \mathrm{~nm}$. As the volume increases, the small ions can distribute further from the macroions, leading to weaker screening and the stronger repulsion. In Case 3, we added salts (equal numbers of counter ions and co-ions) to Case 2 so that the Debye screening length $\lambda$ is similar to that of Case 1. The resultant interaction is almost the same with Case 1, suggesting that the system size dependence can be adjusted by controlling $\lambda$.

\subsection{Repulsive vs. Attractive}

The like-charged colloid particles normally repulsive each other. This is explained by the DLVO theory in terms of the

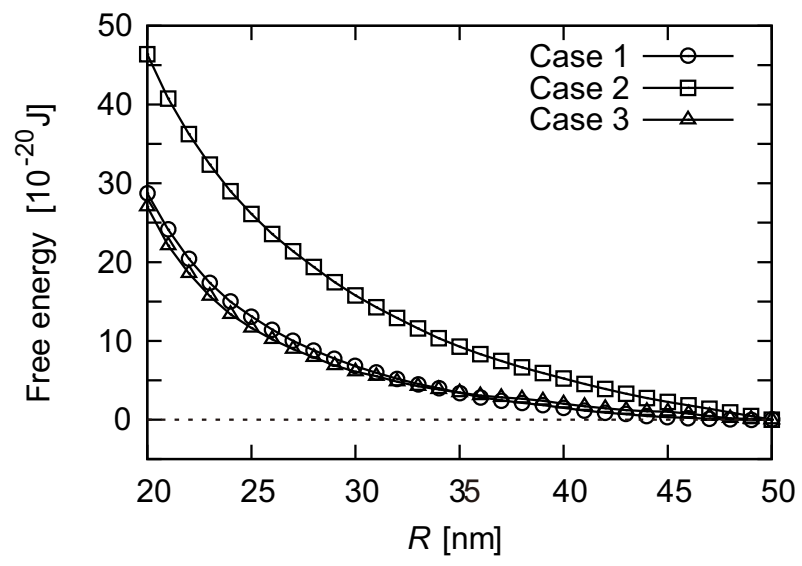

Figure 9: Effective potential for different volumes and different ion concentrations; the conditions are described in Table 2.

overlap of electric double layers. In this theory the electrostatic attraction can not occur. However the electrostatic attraction between like-charged colloid particles at some extreme conditions in some past literatures[12, 13, 14, 15].

The presence of electrostatic attraction with multivalent ions have been found in some articles[12, 13, 14, 15]. In most of these articles the permittivity of solvent is setted lower than that of water to use the strong Coulombic force. Lowering the permittivity of the solvent correponds to the heightening the small ion charge. We varied the counter ion charge as $q=+e$, $+2 e, \ldots$, with keeping other conditions (macroion charge $Z$ and cell volume $V$ ) constant. As shown in Fig. 10, the repulsion is weakened with the increase of $q$; the interaction finally becomes attractive in fictitious cases of $q \geq 10 e$. Although these are salt-free systems, it resembles flocculation or salting-out by counter ions. We observed that counter ions of large $q$ often exist between the two macroions, causing the attractive interaction. This has some resemblance to the recent report[7] with an integral equation method, although the size of the macroions is quite different.

Messina et al propesed the mechanism of the electrostatic attraction with divalent ions[12]. They proposed that macroion charge is lowered by the screening of counter ions. In the case with strong Coulombic force macroion can be overcharged and have reverse sign charge. In two macroion case the state of one overcharged macroion and other undercharged macroion (ionized state) can be metastable and the attraction between two macroion can occur. To investigate the effect of ionized state we change the small ion number valence. As shown in Fig. 11, we simulate three case (a) the left macroion has 8th small ions and right macroion has 12 th smallions, (b) 9 th-11th and (c) 10th10th. In the seperation distance $R=50$, we change the number of small ion which belongs to the left and right macroion. The zero point of free energy is setting to the free energy of $R=20$. In short distance $R$, the effective potential with any case is almost same. This is considered that the small ion can move to other macroion in short distance $R$. In long distance $R$, the free energy is larger as the macroion more ionized. If 


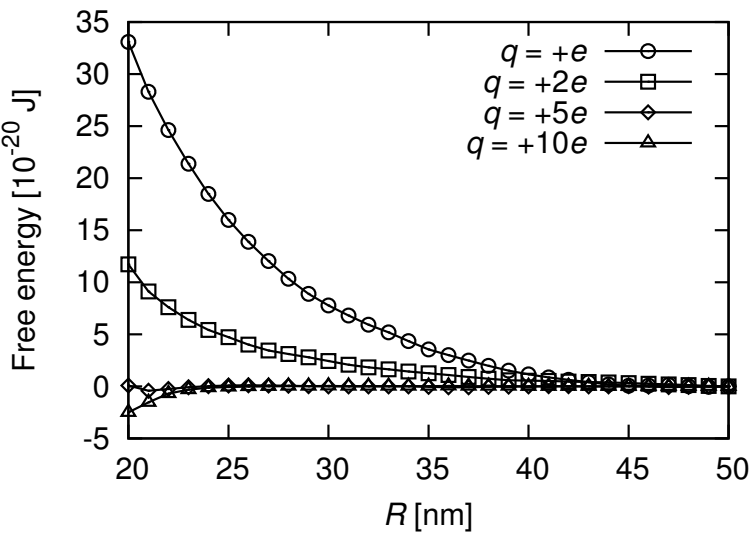

Figure 10: Effective potential for various small ion charges; salt free, $Z=-100$, cell size $100 \mathrm{~nm} \times 50 \mathrm{~nm} \times 50 \mathrm{~nm}$.

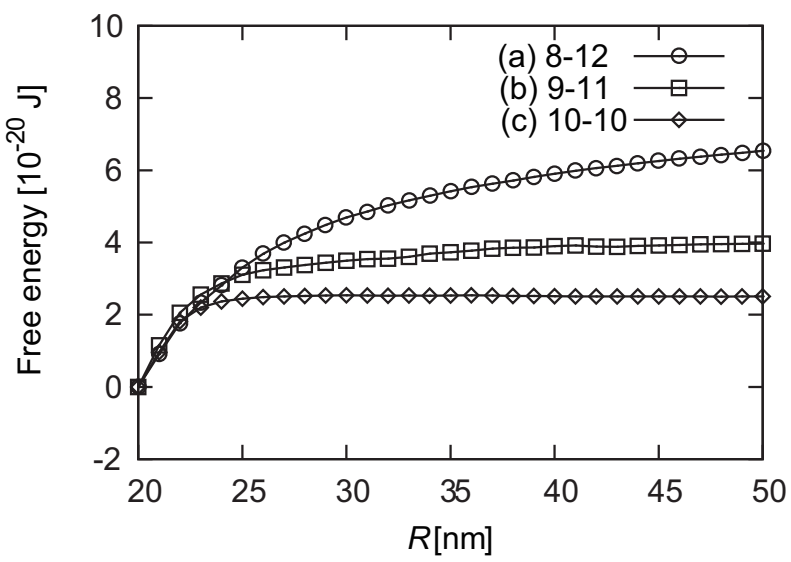

Figure 11: Effective potential for various small ion numbers which are involved in left macroion and right macroion; salt free, $Z=-100$, cell size $100 \mathrm{~nm} \times 50 \mathrm{~nm} \times 50 \mathrm{~nm}, q=+10 e$. Differently from the Fig. 10 free energy $A$ at the $R=20$ is the zero point.

the ionized states exist in the bulk condition, to resolve the imbalance of charge two macroions attract each other and small ion belonging to overcharged macroion move to other undercharged macroion.

For other mechanism Allahyarov proposed the depletion force gained from the imbalance of the counterionic pressure on the macroion[13]. They showed that the depletion force changes from repulsive to attractive if the Coulomb coupling is enhanced. We devide the force acting on the macroion into the Coulombic force and the LJ force to investigate the depletion force because in our simulations LJ force corresponds to the depletion force. From the divided force each effective potential is gained by the thermodynamic integration technique. As showen Fig. 12 for the weak Coulombic case of $q=+e$, the Coulombic repulsion is larger than the LJ repulsion. For the strong Coulombic case of $q=+10 e$ (not ionized state), the Coulombic force and $\mathrm{LJ}$ force become attractive in the short distance $R$. The LJ attraction is stronger than the Coulomic attraction. The LJ force behavior corresponds to the depletion force proposed by Allahyarov et al.
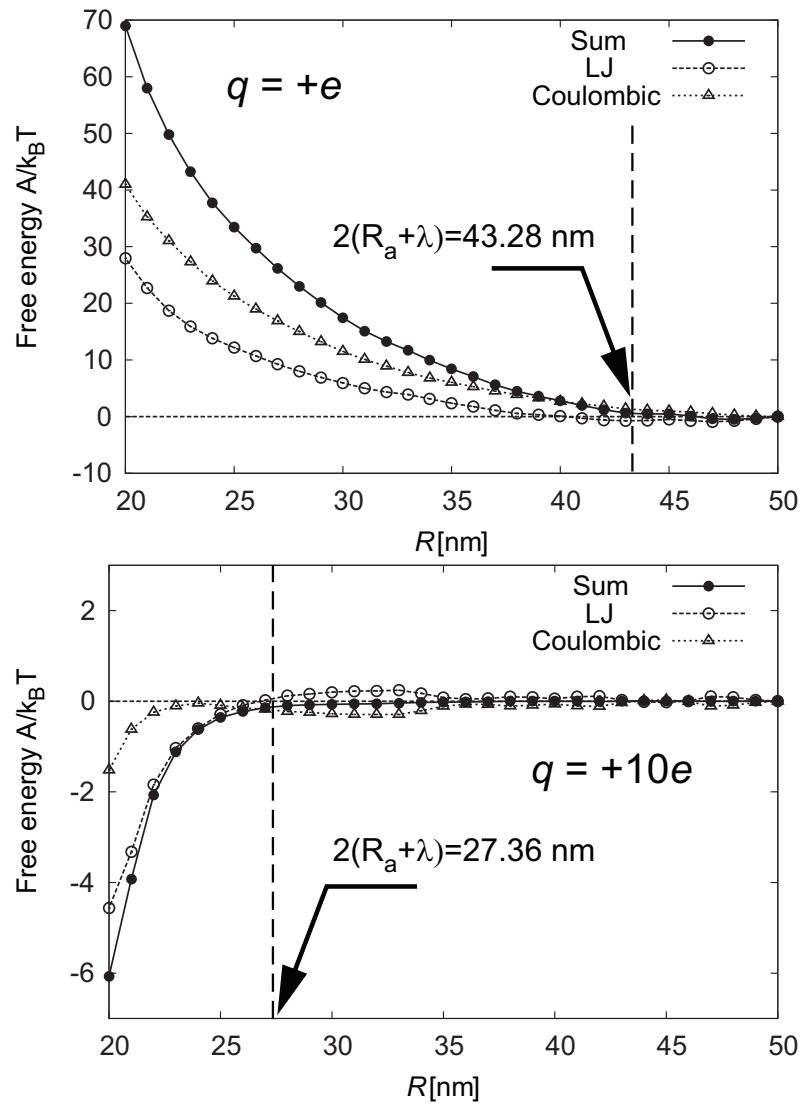

Figure 12: Evaluated effective potential devided into Coulombic potential and LJ potential at the macroion charge $Z=-100$ for a salt-free system in a $100 \mathrm{~nm} \times 50 \mathrm{~nm} \times 50 \mathrm{~nm}$ cell. For the case of $q=+10 e$, the balance of small ion number with macroions is neutral. The Debye screening length is indicated by the vertical dashed line.

\section{Many-body Effect}

In most of theoretical approaches, the effective interaction among macroions is assumed to be sufficiently weak and linearization approximation is made, leading to a sum of two-body interactions.

However three body attraction has been seen by some experiments and some analyses in the some conditions[20, 21, 22, 23]. The common behavior of three body interaction obtained in the past is that when the separation distance $R$ is large, thrre body effect is weak and when the distance $R$ is small, three body effect is attractive except for the contact condition.

In order to investigate the three body effect, we carried out a simulation for a three-macroion system. As shown in Fig. 13, macroions are arranged in an equilateral triangle of side $R$ in the $100 \mathrm{~nm}$ cubic box. Due to the sufficiently bigness of the box, the mean force on each macroion should be directed toward (or from) the triangle center, which can be decomposed into twobody force. By a similar integration described in Sec. 4.1, the effective potential is obtained as a function of distance $R$. The total potential of this system is expressed as follows:

$$
U\left(R_{\mathrm{AB}}, R_{\mathrm{BC}}, R_{\mathrm{CA}}\right)
$$




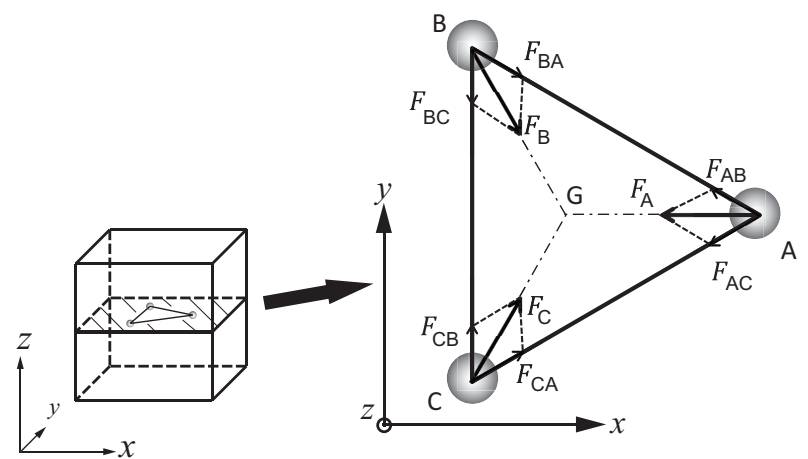

Figure 13: Schematic view of a system with three macroions arranged in an equilateral triangle, the center of which is indicated as $G$. Each mean force $\vec{F}_{\mathrm{A}}$, $\vec{F}_{\mathrm{B}}$, or $\vec{F}_{\mathrm{C}}$ is decomposed into a pair of two-body forces.

$$
\begin{aligned}
= & u_{2}\left(R_{\mathrm{AB}}\right)+u_{2}\left(R_{\mathrm{BC}}\right)+u_{2}\left(R_{\mathrm{CA}}\right) \\
& +u_{3}\left(R_{\mathrm{AB}}, R_{\mathrm{BC}}, R_{\mathrm{CA}}\right)
\end{aligned}
$$

where $u_{2}$ is two body potential and $u_{3}$ is three body potential.

An example is shown in Fig. 14, where the potential for twomacroion case with the same $\lambda$ is also shown for comparison and the difference of effective potential is also showed. The obtained potential is almost same the overlap of the two body interaction in the long distance $R$, and less repulsive in the short distance $R$. So three body interaction is attractive in the short distance beetween macroions as the past articles showing.

\section{Summary}

We carried out molecular dynamics simulations for charged colloidal systems. With the position of colloidal particles (macroions) fixed, the equilibrium distribution of small ions at room temperature was investigated and effective mean force acting on each macroion was evaluated.

In a single macroion system, the charge of the macroion is screened by the counter ions as expected. The screening becomes more complete when the macroion charge increases, and some quantitative deviation from the solution of the linearized Poisson-Boltzmann equation was found. Although the system is highly inhomogeneous, the chemical potential of small ions was constant throughout the system, as expected from the thermodynamic equilibrium conditions.

The effective potential (Helmholtz free energy) between two macroions was evaluated with the thermodynamic integral method. The interaction is repulsive in general, except for some extreme cases where counter ions have large charge. We also found that many-body effect is attractive in the short separation distance.

Except for the extreme cases, we have not found the attractive interaction between macroions, which is suggested in the Sogami model. However this is not conflicting with the Sogami model mainly due to the difference of ensemble. The attractive interaction in the Sogami model is obtained for a constant (osmotic) pressure system, while our simulations were done for a constant volume system. Thus evaluation of the Gibbs free energy, instead of the Helmholtz one, is our next target. We are
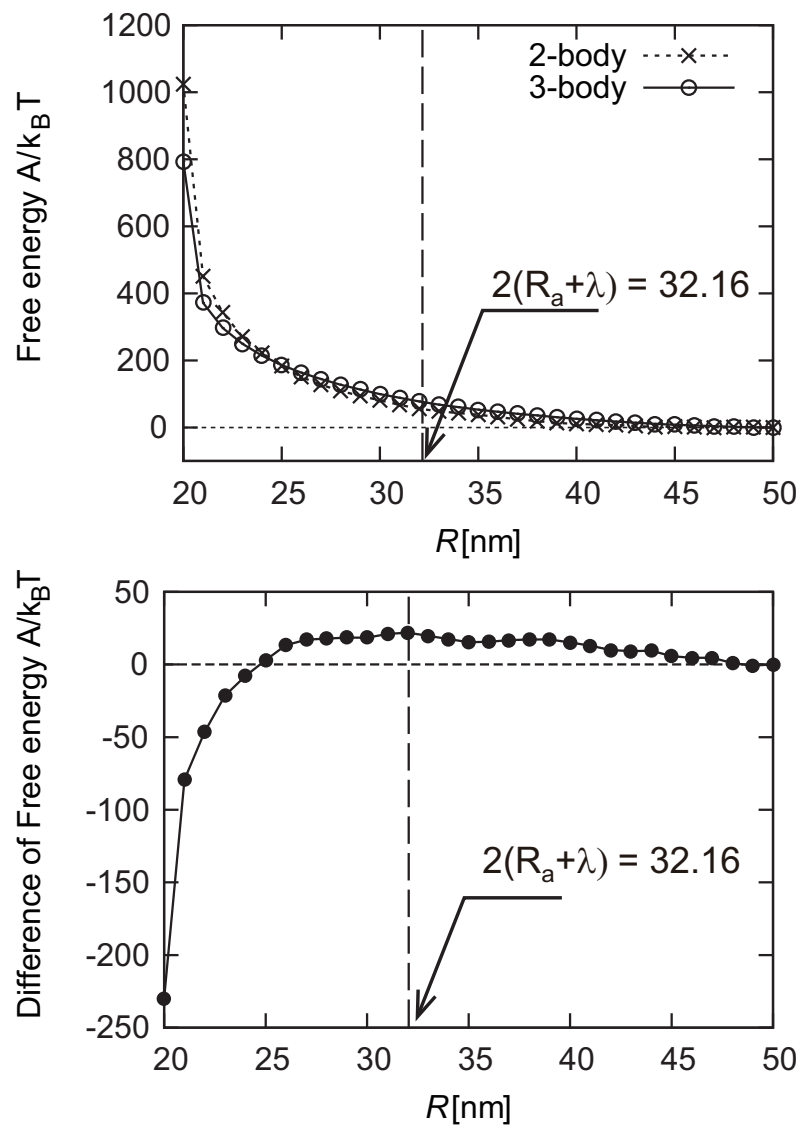

Figure 14: Upper graph shows the effective potential for a triangle arrangement of macroions with $Z=-1000$ in a $100 \mathrm{~nm} \times 100 \mathrm{~nm} \times 100 \mathrm{~nm}$ cell (volume fraction is 0.013), compared with the effective potential for a two-macroion case with the same $\lambda$ and the lower graph shows the difference of effective potential.

planning to do several approaches. One is a simulation with pressure constant conditions; summing up the chemical potential of each species is another.

\section{Acknowledgement}

We are grateful to Prof. I. Sogami (Kyoto Sangyo University), Prof. J. Yamanaka (Nagoya City University) and Prof. M. Ishikawa (RIKEN) for stimulating discussion and encouragement. This work was supported by grants from the Japan Space Forum.

\section{References}

[1] E. J. W. Verwey and J. T. G. Overbeek, Theory of the stability of lyophobic colloids, Elsevier (1948).

[2] B. J. Alder and T. E. Wainwright, Phase transition for a hard sphere system, J. Chem. Phys., 27 (1957) 1208-1209.

[3] B. J. Alder, W. G. Hoover, and D. A. Young, Studies in molecular dynamics. V. High density equation of state and entropy for hard disks and spheres, J. Chem. Phys., 49 (1968) 3688-3696.

[4] S. Hachisu and Y. Kobayashi, Kirkwood-Alder transition in monodisperse latexes II. Aqueous latexes of high electrolyte concentration, J. Coll. Int. Sci., 46 (1974) 470-476. 
[5] I. Sogami and N. Ise, On the electrostatic interaction in macroionic solutions, J. Chem. Phys., 81 (1984) 6320-6332.

[6] N. Ise and I. S. Sogami, Structure Formation in Solution, Springer (2005).

[7] R. Akiyama and R. Sakata, An integral equation study of reentrant behavior in attractive interactions between like-charged macroions immersed in an electrolyte solution, J. Phys. Soc. Jpn., 80 (2011) 123602.

[8] D. L. Ermak and J. A. McCammon, Brownian dynamics with hydrodynamic interactions, J. Chem. Phys., 69 (1978) 1352-1360.

[9] D. J. Adams, Chemical potential of hard-sphere fluids by Monte Carlo methods, Mol. Phys. 78 (1974) 1241-1252.

[10] B. Widom, Potential-distribution theory and the statistical mechanics of fluids, J. Phys. Chem., 86 (1982) 869-872.

[11] M. R. Mruzik, A Monte Carlo study of water clusters, Chem. Phys. Lett., 48 (1977) 171-175.

[12] R. Messina, C. Holm, and K. Kremer, Strong attraction between charged spheres due to metastable ionized states, Phys. Rev. Lett., 85 (2000) 872 875.

[13] E. Allahyarov, I. D'Amico, and H. Löwen, Attraction between likecharged macroions by coulomb depletion, Phys. Rev. Lett., 81 (1998) 1334-1337.

[14] J. Z. Wu, D. Bratko, H. W. Blanch, and J. M. Prausnitz, Monte Carlo simulation for the potential of mean force between ionic colloids in solutions of asymmetric salts, J. Chem. Phys., 111 (1999) 7084-7094.

[15] P. Linse, and V. Lobaskin, Electrostatic attraction and phase separation in solutions of like-charged colloidal particles, Phys. Rev. Lett., 83 (1999) 4208-4211.

[16] J. Yamanaka, S. Hibi, S. Ikeda, and M. Yonese, Particle size dependence for effective charge density of ionic colloids, Molecular Simulation, $\mathbf{3 0}$ (2004) 149-152.

[17] S. Alexander, P. M. Chaikin, P. Grant, G. J. Morales, and P. Pincus, Charge renormalization, osmostic pressure, and bulk modulus of colloidal crystals: Theory, J. Chem. Phys., 80 (1984) 5776-5781.

[18] L. Bocquet, E. Trizac, and M. Aubouy, Effective charge saturation in colloidal suspensions, J. Chem. Phys., 117 (2002) 8138-8152.

[19] P. Wette, H. J. Schöpe, and T. Palberg, Comparison of colloidal effective charges from different experiments, J. Chem. Phys., 116 (2002) 1098110988.

[20] H. Löwen, and E. Allahyarov, The role of effective triplet interactions in charged colloidal suspensions, J. Phys. Cond. Matt., 10 (1998) 4147 4160.

[21] J. Z. Wu, D. Bratko, H. W. Blanch, and J. M. Prausnitz, Effect of threebody forces on the phase behavior of charged colloids, J. Chem. Phys., 113 (2000) 3360-3365.

[22] C. Russ, H. H. von Grünberg, M. Dijkstra, and R. van Roji, Threebody forces between charged colloidal particles, Phys. Rev. E, 66 (2002) 011402-1-011402-12.

[23] C. Russ, M. Dijkstra, R. van Roji, and H. H. von Grünberg, Three-body forces at work: Three-body potentials derived from triplet correlations in colloidal suspensions, Europhys. Lett., 69 (2005) 468-474.

[24] B. Svensson, B. Jönsson, and C. E. Woodward, Monte Carlo simulations of an electric double layer, J. Phys. Chem., 94 (1990) 2105-2113.

[25] M. Aubouy, E. Trizac, and L. Bocquet, Effective charge versus bare charge: an analytical estimate for colloids in the infinite dilution limit, J. Phys. A: Math. Gen., 36 (2003) 5835-5840.

[26] L. Belloni, Ionic condensation and charge renormalization in colloidal suspensions, Colloid Surf. A: Physicochem. Eng. Aspects, 140 (1998) 227-243. 